\title{
Pharmacotherapy of Acute Bipolar Depression in Adults: An Evidence Based Approach
}

\author{
Ather Muneer* \\ Department of Psychiatry, Islamic International Medical College, Riphah International University, Rawalpindi, Pakistan
}

In the majority of cases of bipolar disorder, manic episodes are usually brief and typically responsive to currently available psychopharmacological agents. In contrast, depressive manifestations are more prevalent and persistent, and can present as major depressive/mixed episodes or residual interepisode symptoms. The depressive phase is often associated with other neuropsychiatric conditions, such as anxiety spectrum disorders, substance use disorders, stressor-related disorders, and eating disorders. It is viewed as a systemic disease with associated ailments such as metabolic syndrome, diabetes mellitus, and cardiovascular disease. There is an increased rate of mortality not only from suicide, but also from concomitant physical illness. This scenario is made worse by the fact that depressive symptoms, which represent the main disease burden, are often refractory to existing psychotropic drugs. As such, there is a pressing need for novel agents that are efficacious in acute depressive exacerbations, and also have applicable value in preventing recurrent episodes. The rationale of the present review is to delineate the pharmacotherapy of the depressive phase of bipolar disorder with medications for which there is evidence in the form of observational, open-label, or double-blind randomized controlled studies. In the treatment of acute bipolar depression in adults, a comprehensive appraisal of the extant literature reveals that among mood stabilizers, the most robust proof of efficacy exists for divalproex sodium; while atypical antipsychotics, which include olanzapine, quetiapine, lurasidone, and cariprazine, are also effective, as demonstrated in controlled trials.

Keywords: Bipolar Depression; Drug Therapy; Mood Stabilizers; Anticonvulsants; Atypical Antipsychotics 


\section{INTRODUCTION}

Bipolar disorder (BD) is a collection of heterogeneous conditions; it is conceptualized as a spectrum disorder in the current classifications of Diagnostic and Statistical Manual of Mental Disorders, fifth edition and International Classification of Diseases-10. ${ }^{1)}$ In the former, at least four disorders are recognized: BD type I, in which there is the occurrence of at least one manic or mixed episode in addition to major depressive episodes (MDE); BD type II (hypomanic episodes in addition to MDE); cyclothymic disorder (hypomanic episodes as well as depressive episodes which fall short of MDE); and BD not otherwise specified. In a severe episode, the patient may experience psychotic symptoms and, if these are mood incongruent, the differentiation from schizoaffective disorder and schizophrenia can become difficult. The term 'rapid cycling' is used when a patient suffers from four or more episodes in a 12-month period.

Bipolar spectrum disorders have a high prevalence rate of about $4 \%$ in the general population. ${ }^{2)} \mathrm{BD}$ is a chronic, life-long condition with the usual onset in adolescence or early adulthood. The index episode is usually an MDE with melancholic features, and the first exacerbation may occur several years later in the trajectory of the illness, resulting in an inopportune delay in the correct diagnosis. In symptomatic younger patients, there may be a positive family history of $\mathrm{BD}$, and children who go on to develop the illness often suffer from precursor disorders such as separation anxiety disorder, attention deficit hyperactivity disorder, oppositional defiant disorder, and conduct disorder. ${ }^{3)}$ There is a very high incidence of co-occurring neuropsychiatric conditions, chief among which are substance use and anxiety spectrum disorders. The prognosis of the disease is worsened by the fact that patients can have recurrent and non-remitting depressive episodes, misuse of illicit drugs, and the application of inappropriate and inadequate therapeutic measures, as the diathesis tends to masquerade in myriad different forms. Additionally, the burden of the disorder is further increased by the frequent association of systemic illnesses such as metabolic diseases, cardiovascular disorders, autoimmune diseases, and endocrine disorders. ${ }^{4)}$ Figure 1 schematically depicts these notions and illustrates the pernicious nature of BD (Figure 1).

The bipolar phenotype derives from several individual, inherited, alleles of small effect, thus making it a polygenic disorder. It is a disorder with complex gene-environment interaction in which predisposed individuals suffer from mood episodes, precipitated by stressful events. The course of the disease is virulent in a substantial majority of patients, and may be characterized by poor prognostic features such as rapid cycling, treatment refractoriness, and comorbidities with cognitive and functional decline. Among affected individuals, the depressive phenomena and associated conditions are responsible for the excess morbidity and mortality from diverse psychological and physical factors such as attempted suicide and cardiovascular disease. The de-

\section{Therapeutic implications}

-Treatment refractoriness

-Polypharmacy

-Non-compliance
Neuropsychiatric features

-Non-remission of episodes

-Substance abuse

-Increased suicidality

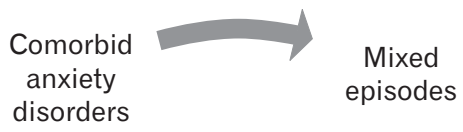

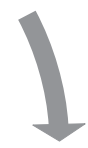

Subthreshold symptoms

Nonremitting depressive symptoms

Physical complications

Functional consequences

-Cognitive impairment

-Psycho-social decline

-Loss of autonomy
Figure 1. In bipolar disorder, affective symptoms act in a vicious cycle causing exacerbation of the illness, leading to adverse biopsychosocial sequelae. 
pressive symptoms are often recalcitrant and show poor response to currently available psychopharmacological agents. ${ }^{5)}$ Antidepressant monotherapy is of little efficacy in bipolar depression, and is perhaps contraindicated because of the liability of causing mood destabilization with a switch into mania or hypomania, and induction of mixed episodes, as well as rapid cycling. ${ }^{6}$ Therefore, the treatment of all phases of BD is primarily with mood stabilizers, which includes first line agents such as lithium, sodium valproate, and carbamazepine. However, the armamentarium has increased in this regard as newer anticonvulsants and atypical antipsychotics are being increasingly employed in the treatment of BD. In addition to mood stabilizing medications, bipolar depression is often responsive to chronotropic interventions, such as single or repeated total sleep deprivation and light therapy. ${ }^{7}$ Figure 2 gives an overview of the present understanding of the biological treatment of bipolar depression.

The current neurobiological perspective of BD dictates that neurotransmitter imbalance is the path through which inherited abnormalities, working in concert with environmental stressors, cause expression of the bipolar phenotype. ${ }^{8)}$ In this vein, modern psychopharmacological agents have neurotransmitter receptors as their primary target, while downstream effects and regulation of gene expression result in long-term changes that are crucial in determining the outcome of the disease. The purpose of the review is to provide an outline of the pharmacological treatments of bipolar depression with agents for which evidence exists in the form of randomized controlled trials, open-label, or observational studies. Only those drugs which have efficacy in this regard, with emphasis on anticonvulsants and atypical antipsychotics, as these are the principal medications employed in the management of this difficult to treat condition, are described here.

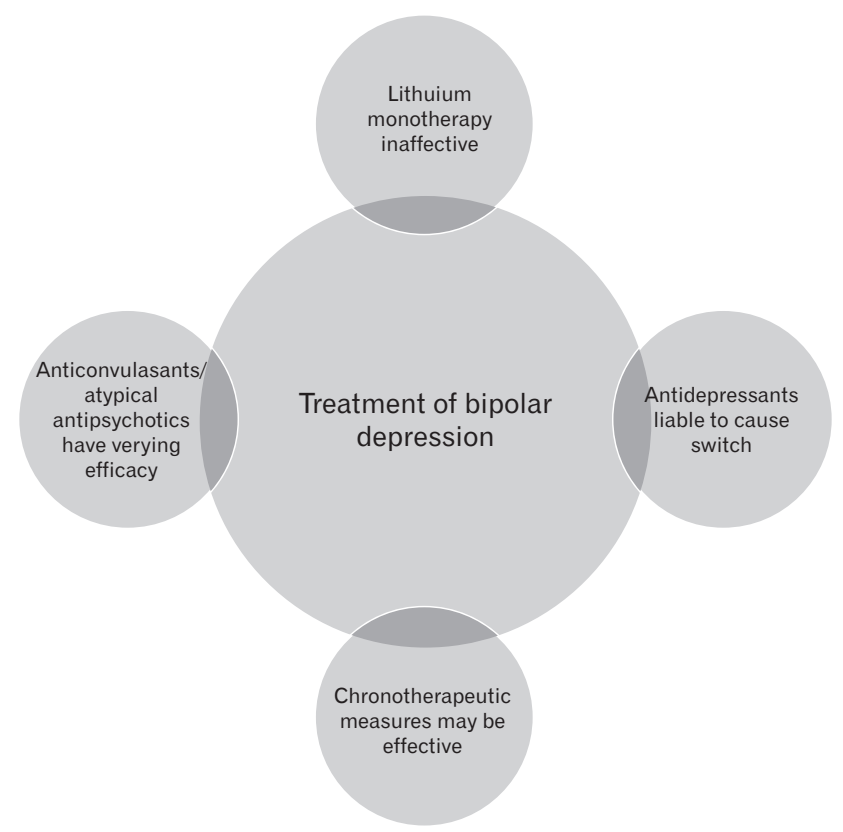

Figure 2. Bipolar depression is difficult to treat with refractoriness to conventional mood stabilizers like lithium.

\section{PRIMARY CARE PERSPECTIVE}

For primary care physicians, the importance of incorporating a mental health viewpoint in the usual care of patients is immense. In this regard, the following points are of particular significance: (1) BD may first present to the family doctor, as the usual age of onset is in adolescence or early adulthood. (2) In youth, BD may present as a melancholic depressive episode and the treating physician may empirically initiate treatment with antidepressants. If the episode is not self-limiting, this may have the impact of worsening the clinical picture by inducing mixed symptoms, or even rapid cycling. (3) Bipolar diathesis is notorious for masquerading in various different ways; examples include substance misuse, disorders of conduct, and presentation as anxiety or panic disorder. If the general practitioner is alert to these possibilities, it may help in arriving at the correct diagnosis and use of appropriate management strategies. (4) BD is a lifelong illness, ultimately, best treated by a mental health specialist. As the overall care of the patient rests with the family physician, it is highly desirable that they are aware of the psychotherapeutic agents employed in the treatment of this disorder, and are able to follow the psychopharmacological strategies employed during the course of the disorder. (5) The primary care physician should be aware that $\mathrm{BD}$ has several associated physical comorbidities, chief among which are metabolic syndrome, diabetes mellitus, and cardiovascular disease. Addressing these is crucial in the overall care of the patient, and better cooperation with the attending psychiatrist is essential to improve the outcome for the patient. (6) Finally, women with BD are a unique population. Special considerations are required during such periods as pregnancy, puerperium, and lactation, and a well-informed family physician can be an invaluable asset for this group of patients.

\section{SEARCH STRATEGY}

In January 2016, the PubMed database was explored with a variety of terms, for example 'bipolar depression and treatment,' 'bipolar depression and lithium,' 'bipolar depression and anticonvulsants, and 'bipolar depression and atypical antipsychotics.' This approach led to the retrieval of more than 1,000 articles. The search was refined by limiting the time period to the last 10 years, and appraisal of the literature was further categorized by activating the filters 'clinical trial', and 'randomized controlled trial,' In addition, studies conducted in children and adolescents were excluded. This approach resulted in less than 100 articles that were pertinent to the current topic. These were read in detail and their reference lists were also consulted. Following this protocol, a consistent representation emerged regarding the pharmacological management of acute bipolar depression in adults, which was organized in a coherent and succinct manner to illuminate the subject under consideration. During the endeavor, a conscious effort was made to objectively bring forth facts that had a firm basis in the scientific literature and this resulted in a wide-ranging evaluation of the efficacy, or otherwise, of psychotropic medications commonly employed in the 
treatment of BD.

\section{LITHIUM AND ANTICONVULSANTS}

\section{Pharmacodynamic Considerations}

Lithium - the prototype mood stabilizer-works, in part, by inhibiting brain glycogen synthase kinase-3 beta (GSK-3 $\beta$ ), which is a serinethreonine kinase. GSK-3 $\beta$ phosphorylates nuclear factor-kappa $B$, heat shock protein factor-1, activator protein-1, and $\beta$-catenin, triggering growth factor cascades. ${ }^{9)}$ Thus, GSK-3 $\beta$ modulates metabolism and survival, the neuronal cytoskeleton, and programmed cell death or apoptosis. Enhanced apoptosis is particularly relevant to the induction of manic symptoms in $\mathrm{BD}$, as demonstrated in transgenic murine models in which the experimental animals showed increased maniclike behaviors in line with augmented GSK-3 $\beta$ activity. ${ }^{10)}$ By downregulating GSK-3 $\beta$, lithium reduces neuronal apoptosis and leads to the amelioration of manic behaviors.

GSK-3 $\beta$ control is also involved in Wnt signaling pathways that regulate development and cell proliferation. Wnt binds to Frizzled receptors present on cellular membranes and subsequently, stimulates a phosphoprotein, the Dishevelled partner, DVL-1, which modulates gene expression through $\beta$-catenin. This pathway, working through Frizzled receptors and DVL-1, can inactivate GSK-3 $\beta$ and lead to normalization of abnormal behaviors, such as increased talking and social withdrawal, seen in different mood states in BD. ${ }^{11)}$ It has been demonstrated that both lithium and anticonvulsant mood stabilizers, like sodium valproate and carbamazepine, regulate GSK-3 $\beta$ activity. ${ }^{12)}$ Additionally, anticonvulsants act through further mechanisms such as inhibition of histone deacetylase, and modulation of astrocyte-associated proteins to promote neurogenesis and enhanced connectivity in key limbic structures like the subiculum and nucleus accumbens. ${ }^{13)}$ Figure 3 gives an illustration of the main concepts in the mood regulating mechanisms of these psychotropic medications. Table 1 provides a selection of studies giving a representation of the therapeutic impact of the agents described in this section.

\section{Lithium Salts}

A literature search revealed that only one placebo-controlled, randomized trial of lithium monotherapy in acute BD type I depression has been published in the last 10 years-the EMBOLDEN I study. In this

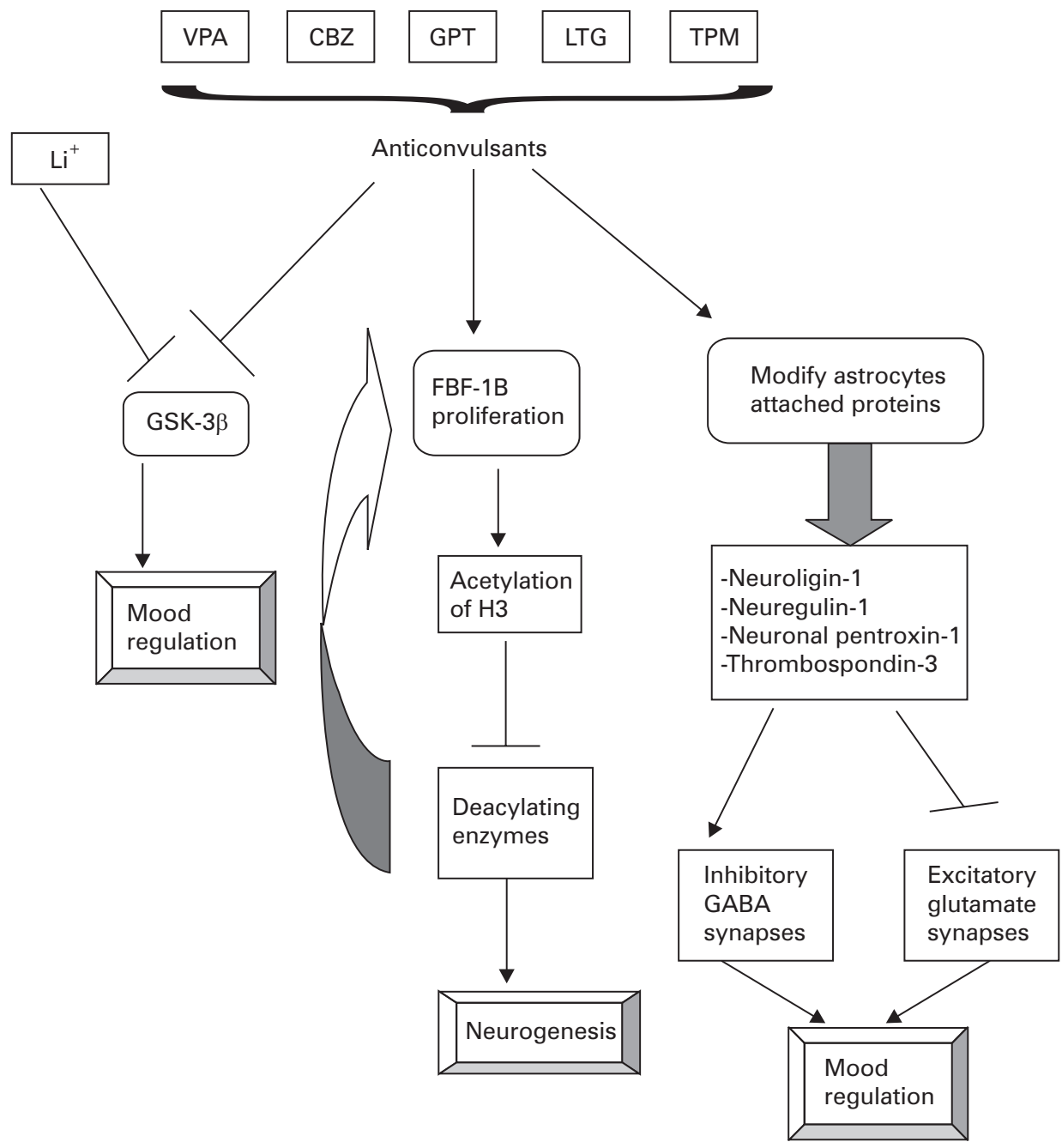

Figure 3. The distributed action of lithium ions and anticonvulsants in the regulation of mood. Anticonvulsant mood stabilizers have a pharmacodynamic advantage over lithium in normalizing abnormal manifestations of mood in bipolar disorder. Much like lithium, these inhibit the enzyme GSK-3 $\beta$, but additionally act through fibroblast growth factors, histone deacetylation, and astrocyte attached proteins to stabilize mood. VPA, valproic acid; CBZ, carbamazepine; GPT, gabapentin; LTG, lamotrigine; TPM, topiramate; Li, lithium; GSK$3 \beta$, glycogen synthase kinase-3 beta; FBF-1B, fibroblast growth factor- $1 \mathrm{~B}$. 
Table 1. $\mathrm{LI}$ and anticonvulsants in the treatment of $\mathrm{BD}$

\begin{tabular}{|c|c|c|c|}
\hline $\begin{array}{l}\text { Psychopharmacological } \\
\text { agent }\end{array}$ & $\begin{array}{l}\text { Type of } \\
\text { research evidence }\end{array}$ & Conclusion & Study \\
\hline $\mathrm{LI}$ & RCT & $\begin{array}{l}\text { LI compared to PLB and quetiapine. LI did not differ significantly from PLB and was inferior } \\
\text { to quetiapine. Conclusion: LI not an effective therapy in acute bipolar depression. }\end{array}$ & Young et al. $(2010)^{14)}$ \\
\hline CBZ & RCT & $\begin{array}{l}\text { CBZ (IR) and (XR) compared in BD patients with MADRS and YMRS scores } \geq 15 \text {. Both } \\
\text { agents equally effective. Conclusion: CBZ effective in both mania and depression. }\end{array}$ & El-Mallakh et al. $(2010)^{17)}$ \\
\hline OXC & RCT & $\begin{array}{l}\text { Study population: BD type I and II patients partially responsive to LI therapy. OXC and CBZ } \\
\text { added adjunctively and patients followed for } 8 \text { weeks. Adjunctive OXC significantly more } \\
\text { effective than CBZ on efficacy measures. Conclusion: OXC a potentially useful therapy in } \\
\text { BD. }\end{array}$ & Juruena et al. (2009) \\
\hline ESC & RCT & $\begin{array}{l}\text { ESC compared to PLB ( } 3 \text { week trial) in bipolar type I patients with manic episodes. ESC no } \\
\text { better than PLB on primary efficacy measure (YMRS). In the continuation phase, ESC } \\
\text { showed global reduction in affective symptomatology with good safety profile. Conclusion: } \\
\text { ESC may have a place in preventing mood episodes in BD. }\end{array}$ & Grunze et al. $(2015)^{19)}$ \\
\hline DV & $\begin{array}{l}\text { Meta-analysis } \\
\text { of RCTs }\end{array}$ & $\begin{array}{l}4 \text { RCTs with a total sample size of } 142 \text { patients. DV significantly superior to PLB in } \\
\text { response and remission rates. Conclusion: DV an effective therapy in acute major } \\
\text { depressive episodes in BD. }\end{array}$ & Bond et al. (2010) \\
\hline LTG & Post-hoc analysis & $\begin{array}{l}\text { Combined analysis of } 5 \text { RCTs of LTG monotherapy versus PLB in acute bipolar depression. } \\
\text { In all trials LTG failed to separate from PLB on main efficacy parameters. Conclusion: LTG } \\
\text { no better than PLB in acute bipolar depression. }\end{array}$ & Calabrese et al. $(2008)^{24)}$ \\
\hline TPM & Open-label study & $\begin{array}{l}\text { Study sample: bipolar type I and II patients with refractory mood symptoms and obesity. } \\
\text { Open-label TPM added adjunctively and patients studied for } 12 \text { weeks. Primary efficacy } \\
\text { measure: CGI, secondary parameters: HRSD-21 and YMRS. Patients showed significant } \\
\text { improvement on all efficacy measures with a reduction of } 2 \text { points in the body mass } \\
\text { index. Conclusion: TPM effective in reducing the severity of mood symptoms and results } \\
\text { in significant weight loss. }\end{array}$ & Gabriel et al. $(2007)^{25)}$ \\
\hline GBP & Open-label study & $\begin{array}{l}\text { Cases receiving first line mood stabilizers with therapeutic plasma levels or atypical } \\
\text { antipsychotics, but with persistent depressive symptoms were included (HRSD-28 scores } \\
\geq 18 \text { ). Open adjunctive GBP added (mean dose } 1,725 \mathrm{mg} / \mathrm{d} \text { ) and patients followed up for } \\
12 \text { weeks. Those with mild to moderate depression had significant improvement, while } \\
\text { the majority of severely depressed subjects did not respond. Conclusion: adjunctive GBP } \\
\text { an efficacious therapy in mild to moderate bipolar depression. }\end{array}$ & Wang et al. (2002) ${ }^{29)}$ \\
\hline PGB & $\begin{array}{l}\text { Open-label } \\
\text { observational study }\end{array}$ & $\begin{array}{l}\text { PGB studied in acute and maintenance periods (2 and } 48 \text { months). Subjects: BD spectrum } \\
\text { outpatients with treatment resistance. Adjunctive PGB given and bipolar psychopathology } \\
\text { measured with CGI-BP. In both parts, significant decrease in symptomatic burden with a } \\
\text { prophylactic effect. PGB well tolerated with no serious drug interactions. Conclusion: in } \\
\text { BD, PGB a safe and effective therapy for acute and recurrent mood episodes. }\end{array}$ & Schaffer et al. $(2013)^{30)}$ \\
\hline LVT & RCT & $\begin{array}{l}\text { A 6-week trial of adjunctive LVT compared to PLB in BD type I or II patients experiencing } \\
\text { acute major depressive episode. Primary efficacy measure: HRSD-21. Secondary } \\
\text { assessments: MADRS, Beck Depression Inventory, Hamilton Anxiety Rating Scale, CGI-BP, } \\
\text { and YMRS. With Last Observation Carried Forward, LVT no better than PLB. Conclusion: } \\
\text { adjunctive LVT not efficacious in acute bipolar depression. }\end{array}$ & Saricicek et al. $(2011)^{32)}$ \\
\hline
\end{tabular}

LI, lithium; BD, bipolar disorder; RCT, randomized controlled trial; PLB, placebo; CBZ, carbamazepine; IR, immediate release; XR, extended release; MADRS, MontgomeryAsberg Depression Rating Scale; YMRS, Young Mania Rating Scale; OXC, oxcarbazepine; ESC, eslicarbazepine; DV, divalproex sodium; LTG, lamotrigine; TPM, topiramate; CGI, Clinical Global Impressions Scale; HRSD, Hamilton Rating Scale for Depression; GBP, gabapentin; PGB, pregabalin; CGI-BP, Clinical Global Impressions Scale-Bipolar Version; LVT, levetiracetam.

trial, patients were treated with either quetiapine 300 or $600 \mathrm{mg} / \mathrm{d}$, lithium 600 to $1,800 \mathrm{mg} / \mathrm{d}$ according to serum levels, or placebo. The duration of the study was 8 weeks and the primary outcome measure was change in Montgomery-Asberg Depression Rating Scale (MADRS) from baseline. The results showed that quetiapine (both doses) was significantly more effective than placebo in reducing MADRS total scores but lithium did not reach statistical significance. Additionally, quetiapine was statistically superior to lithium in treating acute bipolar depression, and that both treatments were equally well tolerated in the short term. ${ }^{14)}$ In this case, there is a need for further controlled lithium studies to better delineate its place in the management of major depressive episodes in BD. Two such trials are ongoing-the ARIQUEL I study (efficacy and safety of the combination of aripiprazole or lithium in bipolar patients with refractory to quetiapine monotherapy) and the comparison of lithium and placebo in suicidal patients in affective disorders. ${ }^{15,16)}$ In conclusion, it can be stated that the current evidence regarding acute bipolar depression is not supportive of lithium monotherapy, but as it is a recalcitrant condition with a substantial suicide risk, adjunctive treatment with this agent may be of value in refractory cases.

\section{Carbamazepine/Oxcarbazepine/Eslicarbazepine}

During the last 10 years no placebo-controlled studies of carbamazepine (CBZ) per se, were found in the extant literature but other work 
has shed light on its role in BD. Importantly, an investigation compared the efficacy and tolerability of immediate-release (IR) and extended-release (ER) CBZ in BD subjects suffering from acute manic, mixed, or depressive episodes. Both BD type I and type II cases were included in the analysis, provided they had MADRS and Young Mania Rating Scale (YMRS) scores $\geq 15$. The patients were already taking CBZ or were considered to benefit from this medication; a comparison of the IR and ER formulations was conducted in a double-blind fashion for the entire study duration of 3 months. There was a statistically significant decrease in the scores of the efficacy measures with both IR and ER carbamazepine. However, the two compositions did not show any significant difference in their outcomes and demonstrated similar adverse effect profiles. ${ }^{17)}$

Oxcarbazepine (OXC) is the keto derivative of CBZ. With respect to bipolar depression, a controlled study of bipolar subjects on lithium monotherapy but with residual symptoms, illuminated its role in BD. Conducted in outpatients, cases with an inadequate response to lithium were randomly assigned to either adjunctive CBZ or OXC in a double-blind manner. Fifty-two cases with lingering manic, mixed, or depressive symptoms as identified using SADS-L (Schedule for Affective Disorders and Schizophrenia-Lifetime Version), were selected and followed up for 8 weeks. Outcome measures included Hamilton Rating Scale for Depression (HRSD)-21, MADRS, YMRS, Clinical Global Impression-Severity (CGI-S), and Clinical Global Impression-Improvement (CGI-I). All subjects completed the study and results were reported for the observed cases. Both CBZ and OXC were statistically significant in producing improvements on the efficacy measures, but OXC showed greater reductions in a range of affective symptoms than CBZ. Additionally, OXC was associated with fewer adverse events and was better tolerated than CBZ. Thus, while there is still a need for adequately powered studies, this preliminary investigation was useful in showing the utility of OXC in $\mathrm{BD}^{18)}$

Eslicarbazepine (ESC), the active enantiomer of metabolized OXC, shares the dibenzazepine ring with its parent compound and CBZ. The drug was approved for epilepsy by the European Medicines Agency in 2009, and since then has been investigated in BD. Presently, it has no approved psychiatric indications, and in a recently published randomized controlled trial (RCT) in acute mania the drug failed to differ from placebo. However, ESC showed valid efficacy on certain outcome measures, in particular CGI-BP (Clinical Global Impression Scale-Bipolar Version), in the long-term. ${ }^{19)}$ While there are ongoing trials, the verdict is still out on the efficacy of this agent in $\mathrm{BD}^{20)}$

\section{Divalproex Sodium}

The anticonvulsant, divalproex sodium (DV), is relatively well studied in adults with acute bipolar depression. In a small preliminary study, nine cases with acute non-refractory bipolar depression were compared with nine control subjects for the trial duration of 6 weeks. The magnitude of reduction in MADRS scores, the primary efficacy measure, was much greater in the treatment arm. Furthermore, a decrease was also observed in YMRS scores, underscoring the efficacy of DV in mixed states. ${ }^{21)}$

In a placebo-controlled RCT, patients with BD type I or II, experiencing major depressive episodes, and not already on a mood stabilizer were randomly assigned to divalproex, extended release, or placebo in a double-blind style. The primary efficacy measure was MADRS and the study duration was 6 weeks. Sixty-seven percent of the subjects met criteria for rapid cycling; statistical evaluation of the entire study population showed that the active compound was significantly better than placebo in inducing response and remission. Sub-group analysis revealed that in BD type II cases, the drug was not significantly different in outcome from placebo, indicating that DV may be more appropriate in acute BD type I depression, particularly in rapid cycling patients. In this short-term study, the drug was associated with gastrointestinal side effects including nausea, increased appetite, and diarrhea. ${ }^{22)}$ In a meta-analysis of four RCTs in acute bipolar depression in adults, DV was found to be superior to placebo with a higher remission rate of $39.3 \%$ versus $17.5 \%{ }^{23)}$

\section{Lamotrigine}

Lamotrigine (LTG) is approved by the United States Food and Drug Administration (FDA) for the maintenance treatment of BD and, in this regard, may have greater value in preventing depressive relapses than manic relapses. With respect to the treatment of acute MDE in bipolar subjects, data from controlled trials reveals that LTG lacks efficacy in this group of patients. A study combined the results of five RCTs in acute bipolar depression, comparing LTG monotherapy with placebo. These trials lasted from 7 to 10 weeks and the active agent was administered at various doses. Three studies used a fixed dose of $200 \mathrm{mg} /$ d. In another study, titration was employed, and subsequently the subjects were given a standard dose of either 50 or $200 \mathrm{mg}$ of LTG per day. In the final study, LTG was flexibly dosed from 100-400 mg daily. HRSD-17 was the primary efficacy measure in two of these trials, while MADRS was employed in the remaining studies. In all five studies, LTG failed to show a significantly different outcome than placebo on the main indicators and in only one trial it showed statistical significance on secondary measures such as CGI-S and CGI-I. Nonetheless, the drug was well tolerated in these acute phase studies. In summary, LTG did not show efficacy in the treatment of acute bipolar depression under controlled conditions. ${ }^{24)}$

\section{Topiramate (TPM)}

A novel antiepileptic used to treat seizures in patients of all age groups, topiramate (TPM) has been studied in BD, particularly in the treatment of acute episodes. Data from RCTs is available in manic and mixed episodes but no placebo-controlled studies for bipolar depression were identified during the literature search. The following conclusions were drawn from the appraisal of the extant literature. ${ }^{25,26)}(1)$ In controlled trials, there is lack of evidence for TPM's efficacy in acute affective episodes. (2) In studies utilizing TPM as an adjunctive agent with primary mood stabilizers and atypical antipsychotics, the drug was well tolerated and no unexpected adverse effects were noted. (3) 
All studies consistently showed a statistically notable reduction in body mass index. (4) TPM could be employed as an add-on agent in therapy with drugs such as olanzapine, which have a high liability of deranging metabolic parameters, perhaps with additional salutary effects on affective symptomatology. (5) It is FDA approved for the prophylactic treatment of migraine, and may be safely used in bipolar subjects with this comorbidity.

\section{Gabapentin/Pregabalin/Gabapentin Enacarbil}

These gamma-aminobutyric acid analogues have the common mechanism of action of binding to the $\alpha 2 \delta$ subunit of voltage-gated calcium channels of presynaptic neurons, decreasing the release of excitatory neurotransmitters and pain mediators. ${ }^{27)}$ Developed as antiepileptic medications, these are extensively used in the treatment of neuropathic pain of various etiologies. BD psychopathology can be considered as comprising of core affective symptoms and attendant neuropsychiatric manifestations, which are invariable concomitants in the majority of patients. Comorbid anxiety worsens the prognosis because of such factors as persistence of symptoms, mixed episodes, substance misuse, and increased suicidality. As such, newer anticonvulsants, including gabapentinoids, are being investigated in bipolar spectrum disorders to provide new perspective in a difficult to treat condition. ${ }^{28)}$ Data from controlled trials are not supportive of the antimanic efficacy of these compounds, and there is a lack of RCTs in acute bipolar depression. There is some information in the form of case reports, observational studies, and prospective trials that show the usefulness of these compounds in acute, as well as maintenance, treatment of $\mathrm{BD}^{29,30)} \mathrm{In}$ summary, gabapentinoids may have value as adjuncts to first line mood stabilizers in refractory patients, and those with anxiety spectrum and substance use disorders as comorbidities. Additionally, these agents may be used in bipolar patients with associated neurological conditions like migraine, neuropathic pain, and restless leg syndrome.

\section{Levetiracetam}

Levetiracetam (LVT) is a broad spectrum antiepileptic used to treat partial epilepsy, with or without secondary generalization, in children as well as adults. It has a novel mechanism of action, exerting its effects by binding to the SV2A synaptic vesicle glycoprotein, inhibiting the P/ $\mathrm{Q}$ type presynaptic calcium channels through an intracellular pathway and resulting in the suppression of glutamate transmission. ${ }^{31)}$ It has been investigated in BD during acute affective exacerbations, and in treatment refractory patients mostly as an adjunctive agent. While there is evidence of efficacy in subjects experiencing manic, mixed, and depressive episodes in non-randomized and open-label trials, this has not been confirmed in RCTs. A single published RCT in acute depression in BD type I and II subjects was conducted with a trial duration of 6 weeks. The primary efficacy measure was HRSD-21 and secondary evaluation was through MADRS, CGI-BP, Beck Depression Inventory, Hamilton Anxiety Rating Scale (HAM-A), and YMRS. Fortytwo cases were randomly assigned to the active agent or placebo; LVT was flexibly administered and the mean dose was 1,132 $\mathrm{mg} / \mathrm{d}$ at endpoint, with the results using a Last Observation Carried Forward (LOCF) method. At endpoint, adjunctive LVT was no better than placebo on primary and secondary outcomes. Under controlled circumstances, add-on LVT was ineffective in treating adult patients with acute bipolar depression. ${ }^{32)}$

\section{ATYPICAL ANTIPSYCHOTICS}

\section{Pharmacodynamic Considerations}

Atypical antipsychotics are a heterogeneous class of medications, having diverse molecular structures but sharing basic mechanisms, which bring about their therapeutic effects. At the dopamine D2 receptor (D2R) level, these medications can be divided into full antagonists, inverse agonists or partial agonists. Risperidone is an example of a full D2R antagonist, olanzapine an inverse agonist, and aripiprazole a partial agonist. Murine models of psychosis suggest that some of these agents act as functionally selective ligands at the D2R, which is a Gprotein coupled receptor (GPCR) ${ }^{33)}$ Downstream signaling occurs through the adenylyl cyclase-cAMP-PKA cascade, and all antipsychotics activate this pathway. However, novel agents like aripiprazole, brexpiprazole, and cariprazine have been shown to selectively work through $\beta$-arrestin 2-Akt-GSK-3 signaling at the GPCR which serves as an alternate downstream pathway with ligand binding at the D2R. ${ }^{34)}$ $\beta$-arrestin 2 is a scaffolding protein which activates protein kinase $\mathrm{C}$ (Akt), phosphorylating the enzyme GSK-3 $\beta$, and ultimately causing

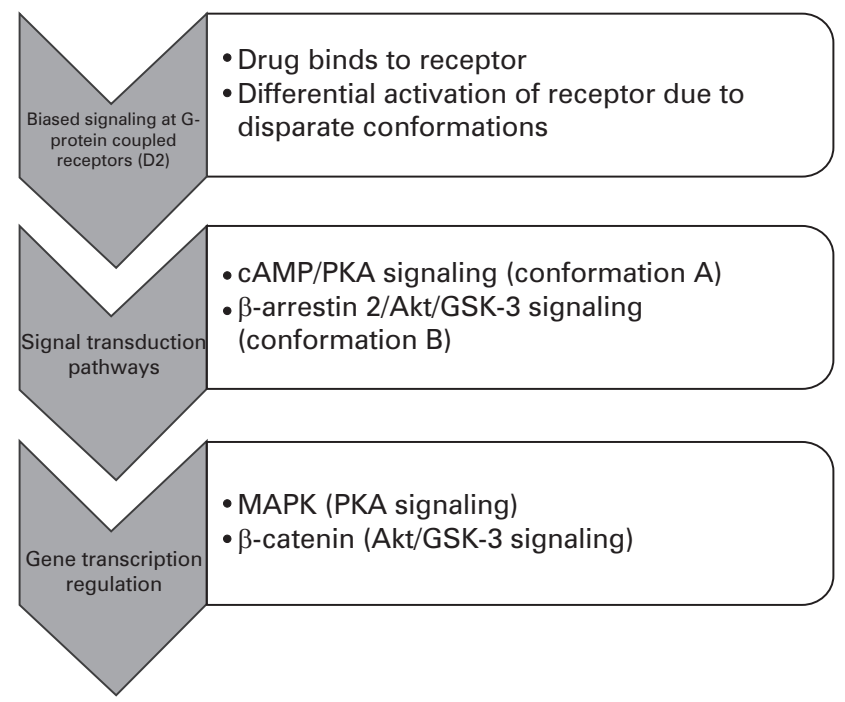

Figure 4. Purported mechanism of biased signaling by novel antipsychotics. Biased ligands are presumed to act in a functionally discriminating manner at G-protein coupled receptors, e.g., D2-type dopamine receptors. One method of functional selectivity may be the favored binding to diverse conformations of the receptor, activating different downstream pathways according to the local milieu and the neuronal sub-types in which these are expressed. Postsynaptic scaffolding proteins, adaptors and effectors may be affected differentially by each receptor conformationrelated cascade differentially stimulated by the ligand. CAMP, cyclic adenosine monophosphate; PKA, protein kinase A; Akt, protein kinase B; GSK-3, glycogen synthase kinase-3; MAPK, mitogen activated protein kinase. 
Table 2. Atypical antipsychotics in the treatment of BD

\begin{tabular}{|c|c|c|c|}
\hline Medication & $\begin{array}{l}\text { Type of } \\
\text { research work }\end{array}$ & Conclusion & Study \\
\hline RLAl & Open-label study & $\begin{array}{l}\text { Subjects: persistently symptomatic BD patients with rapid cycling while on continuing treatment. } \\
\text { Adjunctive RLAl given every } 2 \text { weeks }(25-50 \mathrm{mg} \text { ) for } 16 \text { weeks and change in symptomatology } \\
\text { measured with CGI-BP-S, YMRS, and MADRS. Significant improvement in affective symptomatology at } \\
\text { endpoint, Last Observation Carried Forward. Conclusion: RLAl effective and safe therapy in treatment } \\
\text { refractory bipolar patients. }\end{array}$ & Macfadden et al. (2011) ${ }^{36}$ \\
\hline PDP & RCT & $\begin{array}{l}\text { PDP monthly mono or adjunctive therapy in a 15-month relapse prevention trial in schizoaffective disorder. } \\
\text { Diagnosis with Structured Clinical Interview for Diagnostic and Statistical Manual of Mental Disorders, } \\
\text { fifth edition axis I disorders; primary endpoint time to any relapse: psychotic, manic, or depressive. } \\
\text { Monthly PDP intramuscular superior to PLB in decreasing active mood or psychotic symptoms and } \\
\text { improved functioning. Conclusion: PDP effective in reducing global psychopathology and well tolerated. }\end{array}$ & Fu et al. $(2015)^{37)}$ \\
\hline OZP & Post-hoc analysis & $\begin{array}{l}\text { Pooled analysis of } 2 \text { monotherapy trials in bipolar type I depression. Data stratified with respect to MADRS } \\
\text { total, MADRS-6, and individual item scores (suicidal patients excluded). OZP significantly superior to PLB } \\
\text { on all parameters at week 6. Conclusion: OZP an efficacious therapy for acute major depressive episode } \\
\text { in bipolar I disorder. }\end{array}$ & Tohen et al. $(2013)^{38)}$ \\
\hline OFC & RCT & $\begin{array}{l}\text { Patients with acute bipolar type I depression (MADRS } \geq 20 \text { ) randomized to OZP, OFC, or PLB. Primary } \\
\text { efficacy measure: change in MADRS score at endpoint (week 8). Both OZP and OFC significantly superior } \\
\text { to PLB; OFC statistically better than OZP with higher remission rates. OFC did not cause manic/ } \\
\text { hypomanic switch. Conclusion: OFC more effective than OZP in the treatment of acute bipolar type I } \\
\text { depression. }\end{array}$ & Tohen et al. $(2003)^{39)}$ \\
\hline QTP & $\begin{array}{l}\text { Meta-analysis } \\
\text { of RCTs }\end{array}$ & $\begin{array}{l}\text { Eleven short-term trials included. Majority compared QTP monotherapy with placebo. Participants had } \\
\text { either bipolar type I or II disorders. Primary efficacy measure was change from MADRS score at baseline. } \\
\text { As monotherapy, QTP showed difference from PLB from week } 1 \text { and statistically meaningful difference } \\
\text { maintained until endpoint. Conclusion: QTP an effective and well tolerated treatment in acute bipolar } \\
\text { depression in adults. }\end{array}$ & Suttajit et al. $(2014)^{40)}$ \\
\hline ZRD & Post-hoc analysis & $\begin{array}{l}\text { ZRD monotherapy compared to PLB in two acute } 6 \text {-week trials. Patients (Hamilton Rating Scale for } \\
\text { Depression- } 17 \text { scores } \geq 20 \text { ) randomized to PLB or ZRD (fixed-flexibly dosed from } 40 \text { to } 160 \mathrm{mg} / \mathrm{d} \text { ). } \\
\text { Primary outcome MADRS, mixed-model repeated-measure analysis. In both studies ZRD failed to differ } \\
\text { from PLB on the primary efficacy measure. Conclusion: ZRD no better than PLB in acute bipolar type I } \\
\text { depression. }\end{array}$ & Lombardo et al. $(2012)^{41)}$ \\
\hline LRD & RCT & $\begin{array}{l}\text { Cases with bipolar type I depression randomized to LRD }(20-60 \mathrm{mg} / \mathrm{d}), \mathrm{LRD}(80-120 \mathrm{mg} / \mathrm{d}) \text {, or PLB. } \\
\text { Subjects followed for } 6 \text { weeks, efficacy assessed with MADRS (primary) and CGI-BP (secondary). LRD } \\
\text { (either dose) superior to PLB on both outcomes. LRD therapy associated with minimal metabolic side } \\
\text { effects. Conclusion: LRD monotherapy safe and effective for acute bipolar I depression. }\end{array}$ & Loebel et al. (2014) \\
\hline APZ & RCT & $\begin{array}{l}\text { APZ monotherapy versus PLB in outpatients with non-psychotic, non-suicidal acute bipolar type I } \\
\text { depression. AZP flexibly dosed from 5-30 mg/d. Assessments: MADRS (primary outcome) and CGI-BP-S } \\
\text { (main secondary outcome). At study conclusion (week 8) APZ failed to differ from PLB. Conclusion: AZP } \\
\text { not an efficacious treatment for acute bipolar I depression. }\end{array}$ & Thase et al. (2008) \\
\hline $\mathrm{CPZ}$ & RCT & $\begin{array}{l}\text { Cases with acute bipolar type I depression randomized to PLB or CPZ } 0.75,1.5 \text {, or } 3 \mathrm{mg} / \mathrm{d} \text {. All } 4 \text { groups } \\
\text { assessed with MADRS (primary) and CGI-BP-S (secondary). Analysis with mixed-model, repeated- } \\
\text { measures methodology on the modified intent-to-treat population. Only CPZ } 1.5 \mathrm{mg} / \mathrm{d} \text { achieved } \\
\text { statistical significance at endpoint (week 6). CPZ well tolerated with no serious safety concerns. } \\
\text { Conclusion: CPZ } 1.5 \mathrm{mg} / \mathrm{d} \text { an efficacious and safe treatment for acute bipolar I depression. }\end{array}$ & Durgam et al. $(2015)^{46)}$ \\
\hline
\end{tabular}

BD, bipolar disorder; RLAl, risperidone long-acting injection; CGI-BP-S, Clinical Global Impressions Scale-Bipolar-Severity of Illness Scale; YMRS, Young Mania Rating Scale; MADRS, Montgomery-Asberg Depression Rating Scale; PDP, paliperidone palmitate; RCT, randomized controlled trial; PLB, placebo; OZP, olanzapine; OFC, olanzapinefluoxetine combination; QTP, quetiapine; ZRD, ziprasidone; LRD, lurasidone; CGI-BP, Clinical Global Impressions Scale-Bipolar Version; APZ, aripiprazole; CPZ, cariprazine.

gene transcription via $\beta$-catenin. This cascade appears to control many crucial neuronal functions including expression of receptors and ion conduits, as well as regulation of the process of apoptosis. In affective disorders, there are important implications as key mood regulating areas in the limbic and paralimbic regions are influenced by this signaling. ${ }^{35)}$ Figure 4 schematically illustrates these concepts. Table 2 provides a summation of representative studies of atypical antipsychotics in the treatment of depressive symptoms in $\mathrm{BD}$ and related conditions.

\section{Risperidone}

Risperidone has been well studied in BD, with evidence of efficacy in manic and mixed episodes in BD type I demonstrated in RCTs. No studies of risperidone as mono or adjunctive therapy in acute bipolar depression were found, nonetheless, the value of this drug in the treatment of core affective symptoms was shown in a recently published study. The investigators used a risperidone long-acting injection (RLAI) in symptomatic bipolar patients with rapid cycling. In this open label trial, subjects were marked as having acute depressive or manic/ mixed episodes according to their scores on MADRS and YMRS. Cases with MADRS scores $\geq 16$ were considered as suffering from depressive episodes, while those with YMRS scores $\geq 16$ and any MADRS score were deemed as having manic or mixed episodes. RLAI was given intramuscularly in a dose of 25-50 mg every 2 weeks for the study period 
of 16 weeks. Statistical examination showed that there was significant improvement in CGI-BP and MADRS in depressed subjects, while those with manic and mixed symptoms had notable improvement on YMRS. This analysis called for prospective controlled trials of RLAI in bipolar subjects with acute exacerbations of any polarity. ${ }^{36)}$

Paliperidone or 9-hydroxyrisperidone, the principal metabolite of risperidone, is FDA approved for the treatment of schizophrenia and schizoaffective disorder. In a recently published trial, paliperidone palmitate has shown efficacy in the prevention of psychotic, depressive, and manic symptoms in schizoaffective disorder, but further studies are required to prove its efficacy in $\mathrm{BD}{ }^{37}$ )

\section{Olanzapine}

Olanzapine, a second generation antipsychotic (SGA), has been studied in acute phases of BD. The drug is FDA approved as monotherapy for manic and mixed episodes of BD type I, and its efficacy has also been shown in acute bipolar depression in adults. A post-hoc analysis of two randomized controlled trials demonstrated olanzapine's effectiveness in acute major depressive episodes in BD type I. Olanzapine was employed as monotherapy in a six-week placebo-controlled; 690 patients were randomized to the active agent and 524 to placebo. Data of the study subjects was stratified according to change from baseline in MADRS total score, MADRS-6 sub-score and individual item scores. Further, demographic and disease characteristics, such as psychotic features, melancholic characteristics, and mixed symptoms (defined as scoring $\geq 2$ in $\geq 3$ YMRS items), were also categorized. Analysis of variance with multiple regression analysis showed that, compared to placebo, the olanzapine group had statistically significant score reductions on all efficacy measures except two MADRS items, namely concentration difficulties and suicidal thoughts. The disease factor that best corresponded to better treatment outcome with olanzapine was the presence of melancholic features in the presentation at the start of the study. In summary, olanzapine monotherapy improved key depressive symptoms in BD type $\mathrm{I}^{38)}$ In addition, olanzapine in combination with fluoxetine is FDA approved for the treatment of acute bipolar depression in adults. ${ }^{39)}$

\section{Quetiapine}

Quetiapine, an SGA, is extensively studied in all phases of BD; it is FDA approved for the treatment of manic and mixed episodes of BD type I, and is also licensed as monotherapy for acute bipolar depression. In addition, it is endorsed as a maintenance agent for the prevention of affective episodes as an adjunct to mood stabilizers. A systematic review and meta-analysis was conducted of RCTs of quetiapine in acute bipolar depression, providing an assessment of its efficacy and safety in this condition. The authors conducted an extensive search of different databases including MEDLINE, CINAHL, PsychINFO, etc., leading to the identification of 11 RCTs. The total number of participants was 3,488 with either BD type I or II. Quetiapine was used as monotherapy in the majority of the trials, and compared to placebo or other treatments, such as lithium and selective serotonin reuptake inhibitors.
The primary efficacy measure was change in MADRS scores from baseline to the end of study period. Secondary assessments included CGI-BP-S, CGI-S, HAM-A, Sheehan Disability Scale, Pittsburgh Sleep Quality Index, and Quality of Life and Enjoyment and Satisfaction Questionnaire-Short Form. Quetiapine was significantly more effective than placebo on mean reductions in MADRS total scores, and differences in this regard were observed as early as week 1 . Furthermore, the active agent was also superior on all secondary efficacy measures. In comparison to the other treatments, quetiapine was better than lithium and paroxetine (EMBOLDEN I and II studies) on the chief outcome parameter. In conclusion, quetiapine was an efficacious treatment for acute bipolar depression with a good safety and tolerability profile. $^{40)}$

\section{Ziprasidone}

Ziprasidone, an SGA, has been studied as mono or adjunctive therapy in BD, and is FDA licensed for the treatment of manic and mixed episodes. Two RCTs were conducted comparing ziprasidone alone with placebo in patients with major depressive episodes associated with BD. A cut-off score of 20 was used for HRSD-17 to identify bipolar patients with MDE and cases were randomized to ziprasidone 40 to 160 $\mathrm{mg} / \mathrm{d}$ or placebo and followed for 6 weeks. The principal efficacy parameter was change in MADRS scores at baseline, while secondary measures included HRSD and CGI-I. In both studies, the active agent did not differ significantly from placebo on the main assessment measure. $^{41)}$

Additionally, in another RCT in acute bipolar depression, ziprasidone in combination with a mood stabilizer (lithium, valproate, or lamotrigine) was compared with placebo and mood stabilizer. In this trial too, adjunctive ziprasidone was no better than mood stabilizer alone in patients with bipolar depression. ${ }^{42)}$ This data suggests that ziprasidone does not have efficacy in bipolar depression.

\section{Lurasidone}

Lurasidone, another SGA, is unique in that it has FDA approval for the treatment of acute BD type I, but so far is not licensed for use in manic or mixed episodes. This endorsement is based on two pivotal internationally conducted, randomized, controlled trials in which lurasidone showed superiority over placebo both as monotherapy and as an adjunct to lithium and valproate. Trial duration was 6 weeks; patients had MDE without psychotic features as determined by Mini-International Neuropsychiatric Interview and were treated as outpatients. The primary efficacy measure was reduction in total MADRS score at baseline and key secondary assessment was with the CGI-BP depression severity sub-score. In these trials, lurasidone (flexibly dosed from 20$120 \mathrm{mg} / \mathrm{d}$ ) was superior to placebo on both parameters, showed a good safety profile, and was not associated with significant extrapyramidal side effects or metabolic side effects. ${ }^{43,44)}$ While there is a need for further independent studies corroborating these findings, at this stage lurasidone appears as an important addition to the armamentarium of drugs against bipolar depression. 


\section{Aripiprazole}

Aripiprazole is also referred to as a 'third generation antipsychotic' and shows the pharmacodynamic characteristics of partial agonism, functional selectivity, and serotonin-dopamine activity modulation. It is well studied in different phases of BD and is FDA licensed for manic and mixed episodes of BD type I as monotherapy, and as maintenance treatment in conjunction with a mood stabilizer. Two RCTs of aripiprazole (flexibly dosed from 5-30 mg/d) were conducted in bipolar depression as standalone therapy in comparison to placebo. These were multicenter, 8-week trials of outpatients with non-psychotic bipolar I major depression; the studies were well-powered and utilized change in MADRS total score as the primary outcome measure. CGI-BP depression severity sub-score was the chief secondary assessment parameter; LOCF was used to account for drop-outs. The active agent showed an early difference to placebo treatment, but at week 8 failed to demonstrate statistically significant superiority on both key primary and secondary efficacy measures. Aripiprazole was less well tolerated than placebo, with a higher incidence of akathisia, insomnia, nausea, fatigue, restlessness, and dry mouth. In controlled conditions, aripiprazole was not found to be effective for the treatment of acute depression in BD type I. $^{45)}$

\section{Cariprazine}

Cariprazine, a recently approved novel antipsychotic, is a partial agonist at the D2R/D3R, with higher affinity for the latter receptor. It has already been approved for schizophrenia and the treatment of manic and mixed episodes of BD type I, while studies are ongoing for negative-symptom schizophrenia, major depressive disorder, and bipolar maintenance. In a trial that was recently published online, cariprazine's efficacy was shown in acute BD type I. In this multicenter study, cases were randomized to either cariprazine monotherapy dosed at $0.75,1.5$, or $3 \mathrm{mg} / \mathrm{d}$, or placebo. The primary and secondary efficacy parameters were change from baseline MADRS and CGI-BP scores, respectively, and data were analyzed using a mixed effects model for repeated measures on the adjusted intent-to-treat population. At week 6 , cariprazine $1.5 \mathrm{mg} / \mathrm{d}$ was statistically better than placebo on the primary, as well as secondary, efficacy measures, whereas the other two doses were not significantly different from placebo. The most common adverse events with cariprazine ( $\geq 10 \%$ ) were akathisia and insomnia, while weight gain was slightly higher with active treatment compared to placebo. In this rigorously conducted study, cariprazine monotherapy showed consistent efficacy against bipolar depression and maintained a good tolerability and safety profile. ${ }^{46)}$

\section{CONCLUSION}

$\mathrm{BD}$ is a phasic illness and all current treatments are palliative in nature with varying efficacy. The manic pole of the ailment tends to be of short duration and responsive to the currently existing psychopharmacological agents. In contrast, the depressive phase is often prolonged and poorly controlled by currently available medications. This may express as major depressive episodes, persisting sub-syndromal symptoms, or mixed affective presentations. The high risk of suicide is also attributable to depressive manifestations and, keeping in consideration the associated anxiety and substance use comorbidities, responsible for the major burden of the disease. In this situation, there is an immediate need for better and more efficacious treatments that have an encompassing effect on the diathesis and its myriad complications. In the present review, the foremost psychotropic drugs for bipolar depression have been discussed to inform the modern management of this chronic ailment and evidence-based options are highlighted. It is hoped that with this knowledge, clinicians will be better able to deal with an otherwise intractable condition.

\section{CONFLICT OF INTEREST}

No potential conflict of interest relevant to this article was reported.

\section{REFERENCES}

1. Ghaemi SN. Bipolar spectrum: a review of the concept and a vision for the future. Psychiatry Investig 2013;10:218-24.

2. Ketter TA. Diagnostic features, prevalence, and impact of bipolar disorder. J Clin Psychiatry 2010;71:e14.

3. Nurnberger JI Jr, McInnis M, Reich W, Kastelic E, Wilcox HC, Glowinski A, et al. A high-risk study of bipolar disorder: childhood clinical phenotypes as precursors of major mood disorders. Arch Gen Psychiatry 2011;68:1012-20.

4. Krishnan KR. Psychiatric and medical comorbidities of bipolar disorder. Psychosom Med 2005;67:1-8.

5. Sienaert P, Lambrichts L, Dols A, De Fruyt J. Evidence-based treatment strategies for treatment-resistant bipolar depression: a systematic review. Bipolar Disord 2013;15:61-9.

6. Tondo L, Baldessarini RJ, Vazquez G, Lepri B, Visioli C. Clinical responses to antidepressants among 1036 acutely depressed patients with bipolar or unipolar major affective disorders. Acta Psychiatr Scand 2013;127:355-64.

7. Benedetti F, Barbini B, Fulgosi MC, Colombo C, Dallaspezia S, Pontiggia $\mathrm{A}$, et al. Combined total sleep deprivation and light therapy in the treatment of drug-resistant bipolar depression: acute response and long-term remission rates. J Clin Psychiatry 2005;66:1535-40.

8. Muneer A. Bipolar disorder: role of inflammation and the development of disease biomarkers. Psychiatry Investig 2016;13:18-33.

9. Nery LR, Eltz NS, Martins L, Guerim LD, Pereira TC, Bogo MR, et al. Sustained behavioral effects of lithium exposure during early development in zebrafish: involvement of the Wnt- $\beta$-catenin signaling pathway. Prog Neuropsychopharmacol Biol Psychiatry 2014;55:101-8.

10. Prickaerts J, Moechars D, Cryns K, Lenaerts I, van Craenendonck H, Goris I, et al. Transgenic mice overexpressing glycogen synthase kinase 3beta: a putative model of hyperactivity and mania. J Neurosci 2006;26:9022-9.

11. Watkins CC, Sawa A, Pomper MG. Glia and immune cell signaling in bipolar disorder: insights from neuropharmacology and molecular imaging to clinical application. Transl Psychiatry 2014;4:e350. 
12. Boku S, Nakagawa S, Masuda T, Nishikawa H, Kato A, Takamura N, et al. Valproate recovers the inhibitory effect of dexamethasone on the proliferation of the adult dentate gyrus-derived neural precursor cells via GSK-3 $\beta$ and $\beta$-catenin pathway. Eur J Pharmacol 2014;723:425-30.

13. Wang CC, Chen PS, Hsu CW, Wu SJ, Lin CT, Gean PW. Valproic acid mediates the synaptic excitatory/inhibitory balance through astrocytes--a preliminary study. Prog Neuropsychopharmacol Biol Psychiatry 2012;37:111-20.

14. Young AH, McElroy SL, Bauer M, Philips N, Chang W, Olausson B, et al. A double-blind, placebo-controlled study of quetiapine and lithium monotherapy in adults in the acute phase of bipolar depression (EMBOLDEN I). J Clin Psychiatry 2010;71:150-62.

15. Missio G, Moreno DH, Fernandes F, Bio DS, Soeiro-de-Souza MG, Rodrigues dos Santos D Jr, et al. The ARIQUELI study: potentiation of quetiapine in bipolar I nonresponders with lithium versus aripiprazole. Trials 2013;14:190.

16. Lewitzka U, Jabs B, Fulle M, Holthoff V, Juckel G, Uhl I, et al. Does lithium reduce acute suicidal ideation and behavior?: a protocol for a randomized, placebo-controlled multicenter trial of lithium plus Treatment As Usual (TAU) in patients with suicidal major depressive episode. BMC Psychiatry 2015;15:117.

17. El-Mallakh RS, Salem MR, Chopra A, Mickus GJ, Penagaluri P, Movva R. A blinded, randomized comparison of immediate-release and extended-release carbamazepine capsules in manic and depressed bipolar subjects. Ann Clin Psychiatry 2010;22:3-8.

18. Juruena MF, Ottoni GL, Machado-Vieira R, Carneiro RM, Weingarthner N, Marquardt AR, et al. Bipolar I and II disorder residual symptoms: oxcarbazepine and carbamazepine as add-on treatment to lithium in a double-blind, randomized trial. Prog Neuropsychopharmacol Biol Psychiatry 2009;33:94-9.

19. Grunze H, Kotlik E, Costa R, Nunes T, Falcao A, Almeida L, et al. Assessment of the efficacy and safety of eslicarbazepine acetate in acute mania and prevention of recurrence: experience from multicentre, double-blind, randomised phase II clinical studies in patients with bipolar disorder I. J Affect Disord 2015;174:70-82.

20. Dimitrakopoulos S, Konstantakopoulos G. Pharmacological agents under research for the maintenance treatment in bipolar disorder. Psychiatriki 2015;26:169-80.

21. Ghaemi SN, Gilmer WS, Goldberg JF, Zablotsky B, Kemp DE, Kelley ME, et al. Divalproex in the treatment of acute bipolar depression: a preliminary double-blind, randomized, placebo-controlled pilot study. J Clin Psychiatry 2007;68:1840-4.

22. Muzina DJ, Gao K, Kemp DE, Khalife S, Ganocy SJ, Chan PK, et al. Acute efficacy of divalproex sodium versus placebo in mood stabilizernaive bipolar I or II depression: a double-blind, randomized, placebocontrolled trial. J Clin Psychiatry 2011;72:813-9.

23. Bond DJ, Lam RW, Yatham LN. Divalproex sodium versus placebo in the treatment of acute bipolar depression: a systematic review and meta-analysis. J Affect Disord 2010;124:228-34.

24. Calabrese JR, Huffman RF, White RL, Edwards S, Thompson TR, Ascher JA, et al. Lamotrigine in the acute treatment of bipolar depression: results of five double-blind, placebo-controlled clinical trials. Bipolar Disord 2008;10:323-33.

25. Gabriel A. Adjunctive topiramate treatment in refractory obese bipolar patients: a descriptive open label study. Eat Weight Disord 2007;12:48-
53.

26. Roy Chengappa K, Kupfer DJ, Parepally H, John V, Basu R, Buttenfield J, et al. A placebo-controlled, random-assignment, parallel-group pilot study of adjunctive topiramate for patients with schizoaffective disorder, bipolar type. Bipolar Disord 2007;9:609-17.

27. Kukkar A, Bali A, Singh N, Jaggi AS. Implications and mechanism of action of gabapentin in neuropathic pain. Arch Pharm Res 2013;36: 237-51.

28. Rakofsky JJ, Dunlop BW. Treating nonspecific anxiety and anxiety disorders in patients with bipolar disorder: a review. J Clin Psychiatry 2011;72:81-90.

29. Wang PW, Santosa C, Schumacher M, Winsberg ME, Strong C, Ketter TA. Gabapentin augmentation therapy in bipolar depression. Bipolar Disord 2002;4:296-301.

30. Schaffer LC, Schaffer CB, Miller AR, Manley JL, Piekut JA, Nordahl TE. An open trial of pregabalin as an acute and maintenance adjunctive treatment for outpatients with treatment resistant bipolar disorder. J Affect Disord 2013;147:407-10.

31. Lee CY, Chen CC, Liou HH. Levetiracetam inhibits glutamate transmission through presynaptic P/Q-type calcium channels on the granule cells of the dentate gyrus. Br J Pharmacol 2009;158:1753-62.

32. Saricicek A, Maloney K, Muralidharan A, Ruf B, Blumberg HP, Sanacora $G$, et al. Levetiracetam in the management of bipolar depression: a randomized, double-blind, placebo-controlled trial. J Clin Psychiatry 2011;72:744-50.

33. Peterson SM, Pack TF, Caron MG. Receptor, ligand and transducer contributions to dopamine D2 receptor functional selectivity. PLoS One 2015;10:e0141637.

34. De Bartolomeis A, Tomasetti C, Iasevoli F. Update on the mechanism of action of aripiprazole: translational insights into antipsychotic strategies beyond dopamine receptor antagonism. CNS Drugs 2015;29: 773-99.

35. Park SM, Chen M, Schmerberg CM, Dulman RS, Rodriguiz RM, Caron MG, et al. Effects of $\beta$-arrestin-biased dopamine D2 receptor ligands on schizophrenia-like behavior in hypoglutamatergic mice. Neuropsychopharmacology 2016;41:704-15.

36. Macfadden W, Adler CM, Turkoz I, Haskins JT, Turner N, Alphs L. Adjunctive long-acting risperidone in patients with bipolar disorder who relapse frequently and have active mood symptoms. BMC Psychiatry 2011;11:171.

37. Fu DJ, Turkoz I, Simonson RB, Walling DP, Schooler NR, Lindenmayer JP, et al. Paliperidone palmitate once-monthly reduces risk of relapse of psychotic, depressive, and manic symptoms and maintains functioning in a double-blind, randomized study of schizoaffective disorder. J Clin Psychiatry 2015;76:253-62.

38. Tohen M, Katagiri H, Fujikoshi S, Kanba S. Efficacy of olanzapine monotherapy in acute bipolar depression: a pooled analysis of controlled studies. J Affect Disord 2013;149:196-201.

39. Tohen M, Vieta E, Calabrese J, Ketter TA, Sachs G, Bowden C, et al. Efficacy of olanzapine and olanzapine-fluoxetine combination in the treatment of bipolar I depression. Arch Gen Psychiatry 2003;60:107988.

40. Suttajit S, Srisurapanont M, Maneeton N, Maneeton B. Quetiapine for acute bipolar depression: a systematic review and meta-analysis. Drug Des Devel Ther 2014;8:827-38. 
41. Lombardo I, Sachs G, Kolluri S, Kremer C, Yang R. Two 6-week, randomized, double-blind, placebo-controlled studies of ziprasidone in outpatients with bipolar I depression: did baseline characteristics impact trial outcome? J Clin Psychopharmacol 2012;32:470-8.

42. Sachs GS, Ice KS, Chappell PB, Schwartz JH, Gurtovaya O, Vanderburg DG, et al. Efficacy and safety of adjunctive oral ziprasidone for acute treatment of depression in patients with bipolar I disorder: a randomized, double-blind, placebo-controlled trial. J Clin Psychiatry 2011;72:1413-22.

43. Loebel A, Cucchiaro J, Silva R, Kroger H, Hsu J, Sarma K, et al. Lurasidone monotherapy in the treatment of bipolar I depression: a randomized, double-blind, placebo-controlled study. Am J Psychiatry 2014;171:160-8.
44. Loebel A, Cucchiaro J, Silva R, Kroger H, Sarma K, Xu J, et al. Lurasidone as adjunctive therapy with lithium or valproate for the treatment of bipolar I depression: a randomized, double-blind, placebo-controlled study. Am J Psychiatry 2014;171:169-77.

45. Thase ME, Jonas A, Khan A, Bowden CL, Wu X, McQuade RD, et al. Aripiprazole monotherapy in nonpsychotic bipolar I depression: results of 2 randomized, placebo-controlled studies. J Clin Psychopharmacol 2008;28:13-20.

46. Durgam S, Earley W, Lipschitz A, Guo H, Laszlovszky I, Nemeth G, et al. An 8-week randomized, double-blind, placebo-controlled evaluation of the safety and efficacy of cariprazine in patients with bipolar I depression. Am J Psychiatry 2016;173:271-81. 